\title{
Neuro-ophthalmological biomarkers of visual outcome in newly diagnosed idiopathic intracranial hypertension
}

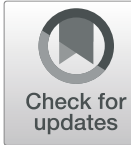

\author{
Wafik Said Bahnasy ${ }^{1,2^{*}}$ (D), Yasser Abo Elfotoh El-Heneedy ${ }^{1,2}$, Mahmoud Ebrahim Mostafa Elhassanien ${ }^{1,2}$, \\ Amr Fawzy Sharaf ${ }^{1,2}$ and Hagar Ahmed Khalid ${ }^{1,2}$
}

\begin{abstract}
Background: Idiopathic intracranial hypertension $(\mathrm{IIH})$ is a disorder of unidentified etiology characterized by raised intracranial pressure (ICP) without clinical, laboratory, or radiological evidence of intracranial pathology. The aim of this work was to determine the visual outcome in newly diagnosed IIH patients.

Methods: The study included $68 \mathrm{IH}$ patients; 59 responded to medical treatment and nine needed lumboperitoneal shunting (LPS). Patients were submitted to papilledema grading using Frisén Scale, water CSF manometry, brain MRI/ MRV, mean deviation of visual field examination (MD-VFE), optic nerve sheath diameter (ONSD), average optic disc optical coherence tomography-retinal nerve fiber layer (OCT-RNFL) thickness, and pattern-reversal visual evoked potential (VEP).
\end{abstract}

Results: Patients needed LPS showed statistically significant increase in baseline papilledema grade, MD-VFE, ONSD, average OCT-RNFL thickness, and P100 VEP latency. On the other hand, both studied groups showed statistically nonsignificant differences regarding the patients' ages and opening CSF pressure.

Conclusion: Newly diagnosed IIH patients' evaluation must be based on multimodality neuro-ophthalmological assessment where papilledema grade, MD-VFE, and OCT-RNFL are valuable biomarkers of PVD while P100 VEP latency delay is a predictor of poor visual outcome and ONSD is an early indicator of elevated ICP regression after LPS surgery.

Keywords: Idiopathic intracranial hypertension, Papilledema, Automated perimetry, Optic nerve sheath diameter, Optical coherence tomography, Visual evoked potential

\section{Introduction}

Idiopathic intracranial hypertension (IIH) is a disorder of unknown etiology characterized by elevation of the intracranial pressure (ICP) without evidences of cerebrospinal fluid (CSF) cytochemical alterations, dilated ventricles, or mass lesions [1]. Its usual clinical presentation includes insidious onset of holo-cranial headache, blurring of vision, pulsatile tinnitus, papilledema, diplopia, and transient visual obscurations (TVO) (brief episodes of orthostatic blurring of vision < $30 \mathrm{~s}$ ) [2]. IIH is more common in obese females during their childbearing age

\footnotetext{
* Correspondence: wafiq.elbahnasi@med.tanta.edu.eg

${ }^{1}$ Department of Neuropsychiatry, Faculty of Medicine, Tanta University, 31527 Tanta, Egypt

${ }^{2}$ Department of Ophthalmology, Faculty of Medicine, Tanta University, 31527 Tanta, Egypt
}

with female:male ratio 5:1. Its incidence is 0.9 per 100,000 general population, 3.5 per 100,000 childbearing women, and 19 per 100,000 childbearing women with $>20 \%$ increase above the ideal body weight [3].

Papilledema is the sine qua none of IIH which is defined as optic disc swelling due to elevated ICP due to impaired axoplasmic flow in the optic nerve $(\mathrm{ON})$ fibers, venous congestion, and fluid leakage [4]. IIH management faces a lot of challenges regarding the best medical treatment, indications of surgery, the ideal time for intervention, model CSF shunting procedure, and when to decide transverse sinus stenting. However, many new drugs are under active trials which will prepare the floor for evidence-based treatment strategies [5]. Dependence on papilledema grading alone during IIH patients' follow-up delays the diagnosis of progressive visual 
deterioration (PVD) and puts $10-20 \%$ of cases at risks of severe irreversible visual impairment. On the other hand, unnecessary CSF diversion modalities put patients at risks of post-operative infections, shunt over-drainage, obstruction, migration, and subsequent failure. So, the introduction of sensitive and objective biomarkers is an essential step to avoid diagnostic delay and ensure better outcome [6].

\section{Aim of the work}

The objectives of this study were to determine the neuro-ophthalmological biomarkers of PVD in newly diagnosed IIH patients and to evaluate the visual outcome of medical as well as surgical management.

\section{Methods}

The present work was a prospective cohort study conducted on $74 \mathrm{IIH}$ patients, recruited from the outpatient clinics of the Neurology and Ophthalmology Departments, Tanta University Hospitals in the period from 1st of July 2017 to the end of November 2018. Six patients were dropped out during the follow-up schedule and the remaining 68 continued the study till its final steps. IIH were diagnosed according to The International Classification of Headache Disorders, 3rd edition (ICHD-3) [7].

Exclusion criteria included patients with recurrent IIH, sinus thrombosis, collagen disorders, or blood diseases. The study's protocol was approved by The Research Ethics Committee and Quality Assurance Unit, Faculty of Medicine, Tanta University. Participations were voluntary, informed consents were obtained from all participants, and the possible risks were clarified.

Baseline initial assessment of the patients was done stressing on the rate of disease progression by estimating the headache onset-papilledema diagnosis time (HPT), presence of TVO or abducent nerve palsy (ANP). Headache severity was evaluated using the headache impact test-6 (HIT-6) and tinnitus was assessed using the Tinnitus Handicap Inventory Severity Scale (THISS). Papilledema was graded using the Frisén Scale and lumbar puncture pressure was done by water CSF manometry. Brain MRI/MRV were done using a 1.5-Tesla, General Electric Scanner (GE Medical Systems, Milwaukee, USA) with quadrature eight channels head coil.

Studied patients were submitted to a battery of neuroophthalmological procedures including automated perimetry visual field examination (VFE) using Humphrey Matrix $^{\circ} 800$, Zeiss software, USA. The mean deviation VFE (MD-VFE) was calculated at 30-2 frequency doubling technology threshold. Non-reliable results with false positive and/or false negative results $>10 \%$ were excluded and/or repeated. Transorbital sonography was done using Ultrasound Philips. Model: HD $11^{\text {ma }}$ XE (Germany), multifrequency $2-5 \mathrm{MHz}$ phased array transducer to measure the optic nerve sheath diameters (ONSD) while the patient lay in recumbent position and close his eyes. The ONSD was assessed $3 \mathrm{~mm}$ behind the lamina cribrosa (after the retroocular bullous end of the $\mathrm{ON}$ ) at which the normative values of the ONSD range is $3.2-5 \mathrm{~mm}$ relied on the work of Kishk et al. 2018 [8].

Spectral domain optical coherence tomography (OCT) was done to exclude pseudo-papilledema and quantity the degree of optic disc elevation by measuring the retinal nerve fiber layer (RNFL) global average thickness using the OCT spectralis, Heidelberg engineering, Heidelberg, Germany. The normative values of OCT-RNFL global average are $91 \pm 7 \mu \mathrm{m}$ regarding the work of Bassi and Mohana 2014 [9] (Fig. 1a). Pattern-reversal visualevoked potential (VEP) was attained using RetiMax device (CSO, Pisa, Italy) at midline occipital electrodes in accordance to the International Society for Clinical Electrophysiology of Vision (ISCEV) to record the N75, P100, and N135 peaks and latencies [10].

Included patients received medical treatment regarding Wall and colleagues 2014 in the form of 1-2 g acetazolamide, low sodium/calories diet, and exercise/weight reduction program. Thirty-four patients needed additional 2-4 therapeutic lumbar CSF taps and nine patients needed neurosurgical consultation. Lumbo-peritoneal shunting (LPS) procedure was considered in patients with progressive papilledema as well as progressive deterioration of the visual field with MD-VFE $\geq-6.5 \mathrm{~dB}$ at any visit or decline $\geq-2.5 \mathrm{~dB}$ from the baseline value in either one or both eyes [11]. The study revealed that 59/68 (86.8\%) patients responded to medical treatment (group I) and 9/68 (13.2\%) patients underwent LPS (group II). All included patients were submitted to baseline and 2-week assessment followed by monthly follow-up neuro-ophthalmological evaluations using the included parameters; papilledema grading, MD-VEP, ONSD, OCT-RNFL global average, and VEP for 6 months to evaluate the visual outcome.

Statistical analysis was conducted using SPSS Prism, version 20, 2013 created by IBM, Illinois, Chicago, USA. Statistical differences between the studied groups were tested using Chi-square for categorical variables and $t$ test as well as ANOVA test for numerical ones. $P$ values $<0.05$ were considered statistically significant.

\section{Results}

Baseline evaluation showed that the mean patients' age was $33.1 \pm 9.5$ years, $62 / 68(91.2 \%)$ were females, $6 / 68$ (8.8\%) were males, BMI was $32.8 \pm 1.7 \mathrm{~kg} / \mathrm{m}^{2}, 13 / 68$ (19.1\%) patients had unilateral ANP, and 24/68 (35.3\%) patients reported TVO. Papilledema grade was $1.99 \pm 0.8$ $(18,37,9$, and 4 patients had grades $1,2,3$, and 4 papilledema respectively). All patients reported mild to 


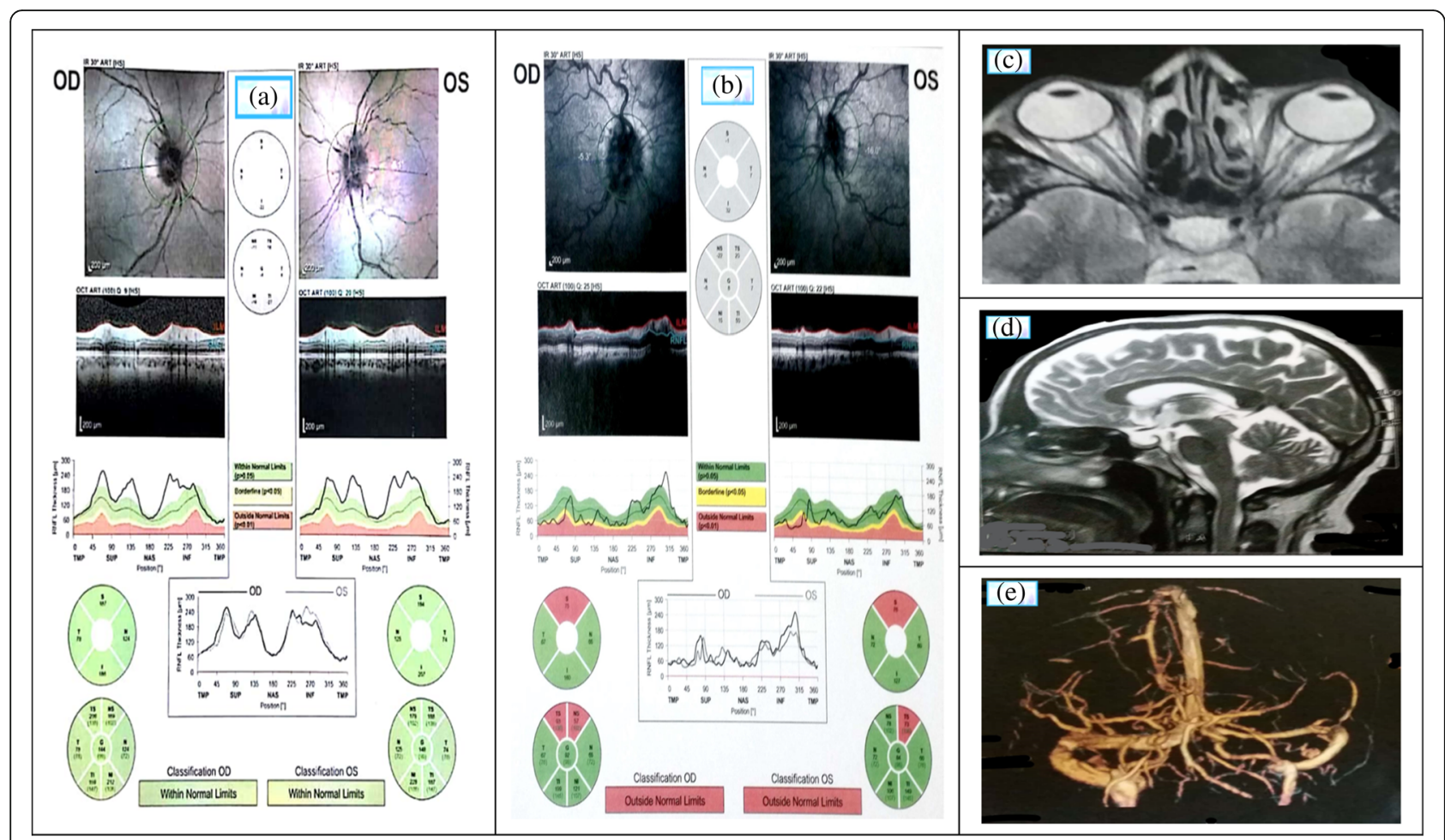

Fig. 1 Optical coherence tomography $(\mathrm{OCT})$ and brain MRI/MRV of IIH cases showing a increased thickness of OCT-RNFL due to papilledema, $\mathbf{b}$ decreased superior RNFL thickness due to axonal loss after 6 months of medical treatments, and $\mathbf{c}-\mathbf{e}$ brain MRI/MRV of IIH patient showing tortuous optic nerves with dilated subarachnoid spaces, empty Sella turcica, and attenuated left transverse sinus

moderate full-cranial headache $(55.9 \pm 3.8$ regarding the HIT-6); the headache onset/diagnosis time was $21.6 \pm$ 6.2 days and $31 / 68(45.6 \%)$ cases reported bilateral mild pulsatile tinnitus $(27.8 \pm 20.8$ regarding the THISS $)$. The baseline CSF pressure was $348.9 \pm 35.5 \mathrm{mmH}_{2} \mathrm{O}$ and the brain MRI/MRV imaging showed that $52 / 68$ (76.5\%) patients had empty sella turcica (EST) and 33/68 (48.5\%) patients had unilaterally attenuated transverse sinus. Regarding the ON, 48/68 (70.6\%) cases showed increased ONSD, tortuosity of the ON, and dilatation of the ON subarachnoid space (Fig. 1c-e). Regarding neuro-ophthalmological assessment, the baseline MD-VEP were $-3.26 \pm 1.27$ and $-3.34 \pm 1.33 \mathrm{~dB}$ and the ONSD were $7.24 \pm 0.68$ and $7.25 \pm 0.67 \mathrm{~mm}$ for the right and left eyes respectively. The average RNFL thickness was $221.9 \pm 56.6$ and $219.8 \pm 57.6 \mu \mathrm{m}$ for the right and left eyes respectively.

The results showed that baseline clinical assessment of patients needed lumbo-peritoneal shunting (group II) showed statistically significant shortening in HPT compared to patients responded to medical treatment (group I). At the same time, group II patients showed statistically significant increase in baseline headache severity (HIT-6) as well as papilledema grade compared to group I patients with $p$ values $<0.05$. Regarding the studied neuro-ophthalmological parameters, group II patients showed statistically significant increase in each of MDVFE, ONSD, and average OCT-RNFL thickness as well as prolongation and P100 VEP latency compared to group I patients with $p$ values $<0.05$. At 6 -month assessment visit, group I showed statistically significant increase in weight reduction than group II (Table 1). On the other hand, both studied groups showed statistically non-significant differences regarding the patients' ages, baseline BMI, THISS, opening CSF pressure, and P100 VEP amplitude as well as the percent of existence of ANP, TVO, EST, and transverse sinus stenosis with $p$ values $>0.05$ (Table 1 ).

Six included patients were children with mean age $13.4 \pm 0.9$ years, BMI $32.6 \pm 1.5 \mathrm{~kg} / \mathrm{m}^{2}, 4 / 6(66.7 \%)$ were males, and $2 / 6$ (33.3\%) were females. Five cases (83.3\%) had unilateral ANP and CSF manometry $344.5 \pm$ $46.3 \mathrm{mmH}_{2} \mathrm{O}$.

The study showed that progressive increase in papilledema grade and OCT-RNFL thickness as well as reduction in MD-VEP and P100 VEP latency delay despite medical treatment were indicators of LPS while the ONSD was the first parameters showed significant regression after surgery (within few days) (Table 2).

The range of LPS procedure timing was 18-35 (26 \pm 6.1) days and patients passed stationary/regressive courses till 35 days from baseline assessment did not 
Table 1 Comparison between medically responded IIH patients (group I) and those who needed lumbo-peritoneal shunts (group II) at initial baseline assessment visit

\begin{tabular}{|c|c|c|c|c|}
\hline & & $\begin{array}{l}\text { Group I } \\
\text { (n: 59) }\end{array}$ & $\begin{array}{l}\text { Group II } \\
(n: 9)\end{array}$ & $p$ vale \\
\hline \multicolumn{2}{|l|}{ Age (years) } & $32.72 \pm 9.94$ & $35.97 \pm 5.83$ & 0.3449 \\
\hline \multicolumn{2}{|c|}{ Body mass index $\left(\mathrm{kg} / \mathrm{m}^{2}\right)$} & $32.76 \pm 1.71$ & $33.24 \pm 1.55$ & 0.425 \\
\hline \multicolumn{2}{|c|}{ Weight reduction after 6-months $(\mathrm{kg})$} & $14.6 \pm 4.2$ & $4.4 \pm 1.4$ & $<0.0001^{*}$ \\
\hline \multicolumn{2}{|c|}{ Headache onset/diagnosis time (days) } & $23.58 \pm 3.62$ & $8.78 \pm 3.15$ & $<0.0001^{*}$ \\
\hline \multicolumn{2}{|l|}{ Headache severity } & $55.37 \pm 3.33$ & $61.11 \pm 3.37$ & $<0.0001^{*}$ \\
\hline \multicolumn{2}{|l|}{ Tinnitus severity } & $25.89 \pm 20.78$ & $40.00 \pm 17.41$ & 0.057 \\
\hline \multirow[t]{5}{*}{ Papilledema grade } & Mean \pm SD & $1.8 \pm 0.64$ & $3.2 \pm 0.67$ & $<0.0001^{*}$ \\
\hline & Grade-I & $18(\%)$ & 0 & $<0.0001^{*}$ \\
\hline & Grade-II & $36(\%)$ & $1(\%)$ & \\
\hline & Grade-III & $4(\%)$ & $5(\%)$ & \\
\hline & Grade-IV & $1(\%)$ & $3(\%)$ & \\
\hline \multicolumn{2}{|c|}{ Opening CSF pressure } & $350.49 \pm 36.61$ & $351.00 \pm 35.27$ & 0.969 \\
\hline \multicolumn{2}{|c|}{ Transient visual obscuration $n(\%)$} & $19(32.2 \%)$ & $5(55.6 \%)$ & 0.172 \\
\hline \multicolumn{2}{|c|}{ Abducent nerve palsy $n(\%)$} & $10(16.9 \%)$ & $3(33.3 \%)$ & 0.244 \\
\hline \multicolumn{2}{|c|}{ Empty sella turcica $n(\%)$} & $45(76.3 \%)$ & $7(77.8 \%)$ & 0.92 \\
\hline \multicolumn{2}{|c|}{ Transverse sinus stenosis $n(\%)$} & $27(45.8 \%)$ & $6(66.7)$ & 0.243 \\
\hline \multirow[t]{2}{*}{ MD-VFE } & Right (decibel) & $-2.9 \pm 0.6$ & $-3.92 \pm 0.44$ & $<0.0001^{*}$ \\
\hline & Left (decibel) & $-2.7 \pm 0.47$ & $-3.97 \pm 0.48$ & $<0.0001^{*}$ \\
\hline \multirow[t]{2}{*}{ ONSD } & Right (mm) & $7.15 \pm 0.64$ & $7.81 \pm 0.62$ & $0.0038^{*}$ \\
\hline & Left (mm) & $7.16 \pm 0.64$ & $7.82 \pm 0.59$ & $0.0052^{*}$ \\
\hline \multirow[t]{2}{*}{ OCT-RNFL } & Right average $(\mu \mathrm{m})$ & $211.66 \pm 53.4$ & $288.7 \pm 18.25$ & $<0.0001^{*}$ \\
\hline & Left average ( $\mu \mathrm{m})$ & $209.19 \pm 54.03$ & $289.19 \pm 18.73$ & $<0.0001^{*}$ \\
\hline \multirow[t]{4}{*}{ VEP } & Right P100 amplitude $(\mu \mathrm{V})$ & $11.7 \pm 3.8$ & $9.8 \pm 2.3$ & 0.15 \\
\hline & Left P 100 amplitude $(\mu \bigvee)$ & $11.2 \pm 3.4$ & $9.6 \pm 2.7$ & 0.18 \\
\hline & Right P100 latency (ms) & $92.78 \pm 6.46$ & $127.3 \pm 7.5$ & $<0.0001^{*}$ \\
\hline & Left P 100 latency (ms) & $94.13 \pm 6.81$ & $127.7 \pm 7.2$ & $<0.0001^{*}$ \\
\hline
\end{tabular}

*: significant; MD-VFE mean deviation of visual field examination, OCT-RNFL optical coherence tomography-retinal nerve fiber layer, ONSD optic nerve sheath diameter, VEP visual evoked potential

Table 2 Follow-up neuro-ophthalmological assessments for progressive IIH patients who needed lumpo-peritoneal shunts (group II)

\begin{tabular}{|c|c|c|c|c|c|c|}
\hline & & \multirow[t]{2}{*}{ Baseline } & \multirow[t]{2}{*}{ 2-week visit } & \multirow{2}{*}{$\begin{array}{l}\text { Post- } \\
\text { operative }\end{array}$} & \multicolumn{2}{|l|}{ ANOVA } \\
\hline & & & & & $f$ value & $p$ value \\
\hline \multirow[t]{2}{*}{ Papilledema grade } & Right & $2.44 \pm 0.73$ & $2.89 \pm 0.78$ & $2.16 \pm 0.53$ & 1.0309 & 0.372 \\
\hline & Left & $2.41 \pm 0.76$ & $3.01 \pm 0.72$ & $2.09 \pm 0.61$ & 1.0289 & 0.301 \\
\hline \multirow[t]{2}{*}{ MD-VFE } & Right (decibel) & $-3.92 \pm 0.44$ & $-6.23 \pm 0.24$ & $-4.11 \pm 0.37$ & 94.360 & $0.0001^{*}$ \\
\hline & Left (decibel) & $-3.97 \pm 0.48$ & $-6.39 \pm 0.29$ & $-4.12 \pm 0.46$ & 114.402 & $0.0001^{*}$ \\
\hline \multirow[t]{2}{*}{ ONSD } & Right (mm) & $7.81 \pm 0.62$ & $8.17 \pm 0.63$ & $4.91 \pm 0.59$ & 0.128 & 0.88 \\
\hline & Left (mm) & $7.82 \pm 0.59$ & $7.96 \pm 0.67$ & $4.87 \pm 0.51$ & 0.825 & 0.45 \\
\hline \multirow[t]{2}{*}{ OCT-RNFL } & Right average $(\mu \mathrm{m})$ & $288.7 \pm 18.25$ & $317.81 \pm 8.53$ & $311.13 \pm 15.41$ & 9.759 & $0.0008^{*}$ \\
\hline & Left average ( $\mu \mathrm{m})$ & $289.19 \pm 18.73$ & $316.34 \pm 9.71$ & $312.43 \pm 15.53$ & 8.475 & $0.0016^{*}$ \\
\hline \multirow[t]{2}{*}{ VEP } & Right P100 latency (ms) & $127.3 \pm 7.5$ & $126.4 \pm 6.5$ & $126.8 \pm 5.7$ & 0.054 & 0.95 \\
\hline & Left P 100 latency (ms) & $127.7 \pm 7.2$ & $127.3 \pm 6.5$ & $125.8 \pm 5.6$ & 0.21 & 0.81 \\
\hline
\end{tabular}


develop subsequent PVD in later visits. In the last 6-month follow-up visit, six patients from group I and three patients from group II showed decrease in average OCT-RNFL thickness (Fig. 1b).

\section{Discussion}

The study revealed that a high proportion of IIH patients responded to medical treatment including weight reduction program with or without lumbar puncture and only $13.2 \%$ needed LPS. Patients needed neurosurgical intervention experienced rapid disease progression with short HPT (fulminant IIH). This finding is passing with that of Hoffmann and colleagues 2018 [12] and Mulroy and colleagues 2018 [13] who stated that most $\mathrm{IIH}$ patients have a relatively benign, self-limiting course except in those with rapid progressive course and fast diminution of vision who carry worse visual outcomes and necessitate rapid CSF shunting.

The study showed that the presence of ANP, TVO, or very high opening CSF pressure during baseline assessment are not indicators of surgery and a big proportion of them underwent subsequent regressive course. These results are passing with the studies of Agarwal and colleagues 2017 [2] and Berezovsky and colleagues 2017 [14] who stated that ANP, TVO, and high CSF pressure are not predictors of poor visual outcome in IIH, but follow-up of cases is needed to diagnose progressive cases.

The study declared different vulnerability of the ON to external compression by the elevated ICP evidenced by the non-significant correlations between CSF pressure and each of papilledema grade, MD-VFE, average OCTRNFL thickness, and asymmetry of these parameters in a sector of patients (Fig. 2). These results are in harmony with that of Markey and colleagues 2016 [15] who stated that the grades of papilledema are sometimes not proportional with the degrees of visual impairments which makes it an imprecise parameter to follow-up IIH patients.

The results of this study showed that obesity is an important IIH predisposing factor especially in post-pubertal females and weight reduction is an effective disease-modifying factor that helps in treatment IIH. Fahmy and colleagues 2016 [16] and Agarwal and colleagues 2017 [2] are keeping with these results and stated that IIH might exhibit high intra-abdominal pressures due to increased visceral obesity which may be transmitted intracranially in the presence of incompetent internal jugular vein valve. By contrast to these results, Biousse 2012 [17] stated that the degrees of obesity are not associated with higher incidence of PVD, but he considered that higher degree of obesity is a predictor of subsequent visual

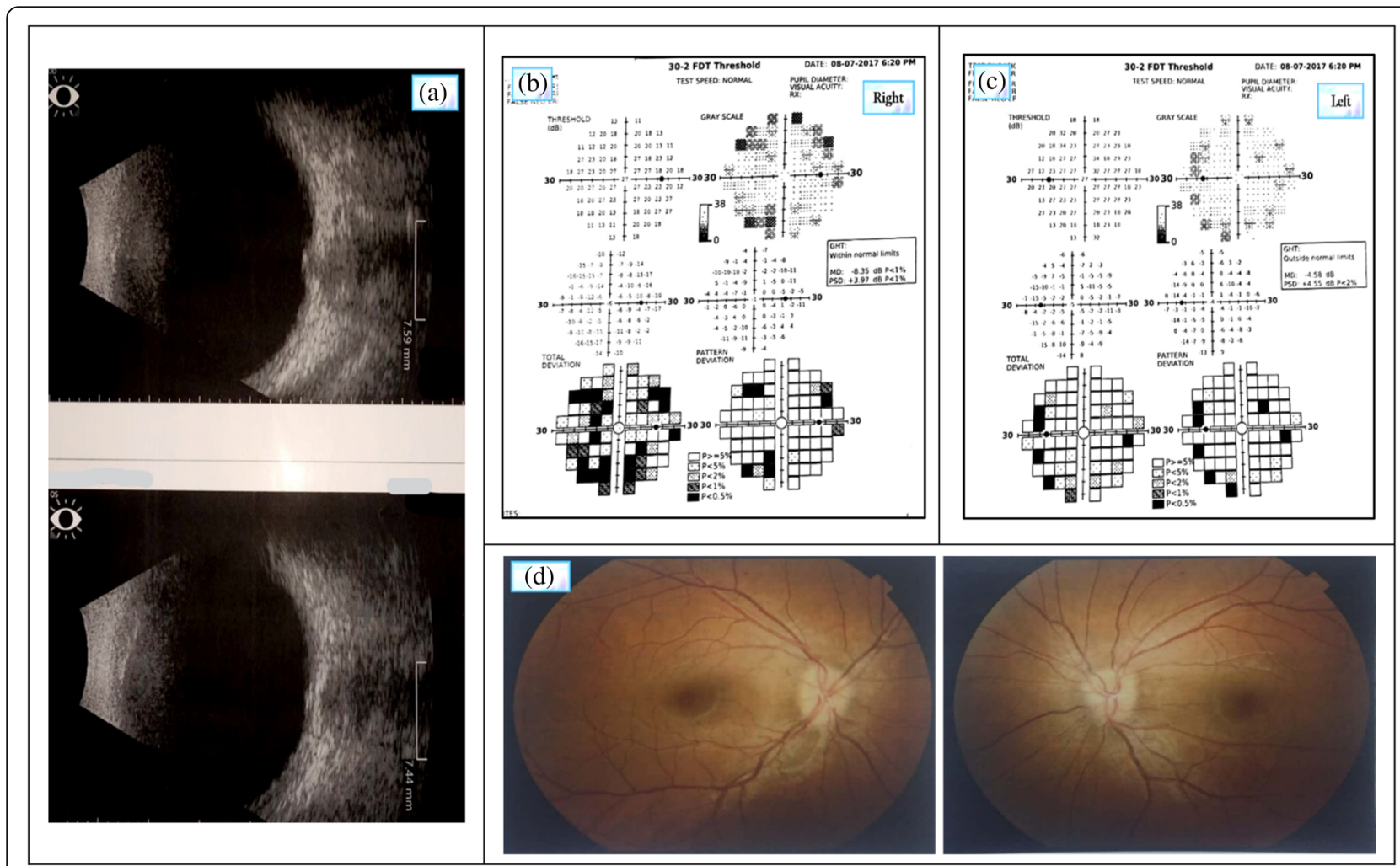

Fig. 2 Baseline ophthalmological assessment of an IIH patient showing a dilated right and left optic nerve sheath diameter (7.59 and $7.44 \mathrm{~mm}$ respectively), $\mathbf{b}, \mathbf{c}$ asymmetrical visual field affection with decreased mean deviation of the automated visual field examination of right and left eyes $(-8.35$ and -4.58 respectively), and $\mathbf{d}$ fundus color photography showing bilateral grade III papilledema 
decline possibly due to higher incidence of obstructive sleep apnea among his studied patients.

The study revealed that brain MRI/MRV of IIH are commonly associated with EST, ON changes (increased ONSD, dilated ON subarachnoid space, and ON tortuosity) and/or transverse sinus stenosis, but these findings are not correlated with the opening CSF pressure and are not predictors of possible subsequent progression or need of LPS. These findings are in harmony with the results of Agarwal and colleagues 2017 [2] who stated that brain MRI/MRV increases the diagnostic certainty of IIH and aid in exclusion of mass lesions, hydrocephalus, and venous sinus thrombosis but are not associated with worse prognosis.

The study showed that childhood onset IIH usually has a relatively benign course and little possibility of progression as all included children had responded to medical treatment. At the same time, there was non-significant sex difference childhood onset IIH which points to the role of gonadal female sex hormones in the pathogenesis of IIH during subsequent pubertal and childbearing periods. This assumption is in harmony with the work of Margeta and colleagues 2015 [18] and Galindo and colleagues 2017 [19] who stated that prepubertal IIH usually has non-significant sex difference, carries a favorable outcome, and infrequent need for surgical intervention.

Transorbital sonographic assessment of the ONSD is an easy, cost-effective non-invasive technique useful in detecting raised ICP [8]. The study showed that ONSD dilatation is a reliable confirmatory diagnostic test for raised ICP due to IIH but has a weak prognostic value as the degree of ONSD was not correlated with the degree of papilledema, and visual field affection as well as possible subsequent PVD. On the other hand, the test is a suitable easy tool to assess post-operative IIH regression and follow-up for possible shunt obstruction as it is the first parameters affected by changes in ICP. These results are in accordance with that of Bekerman and colleagues 2016 [20] who stated that ONSD is a useful test in diagnosis of high ICP, but it needs other additional investigations to quantify its severity. On the contrary, Liu and colleagues 2017 [21] stated that the dilatation of ONSD is proportional with the ICP rise but this difference in results is explained by the inclusion of cases with acute ICP rise due to head trauma or following intracranial surgery rather than gradual elevation by IIH.

Spectral domain OCT is a quick non-invasive technique which uses interferometric analysis of shortcoherence-length infrared light to provide depth-resolved imaging of ocular tissues including the choroid, retina, and ON head [22]. The study showed that spectral domain optical coherence tomography (SD-OCT) is an objective, easy, non-invasive confirmatory tool for diagnosis of early papilledema due to IIH and exclude pseudo-papilledema and $\mathrm{ON}$ drusen. These results are passing with that of Huang-Link and colleagues 2015 [23] and Markey and colleagues 2016 [15] who stated that SD-OCT is a highly sensitive tool to measure the $\mathrm{ON}$ head changes due to IIH including the cup volume, cup/disc ratio, and RNFL thickness. This high accuracy and lower inter-rater reliability make OCT an objective tool to govern the response to treatments and predict possible progression.

The results declared that prolongation of the P100 VEP latency during baseline assessment is a bad prognostic sign and could be considered as a biomarker for subsequent need of LPS. These results are in existence with that of Hartmann and colleagues 2015 [24] who determined that papilledema due to IIH may be associated with some degrees of $\mathrm{ON}$ fibers demyelination and subsequent prolongation of the P100 VEP latency which is associated with poor visual outcome.

The study showed that after resolution of papilledema, some patients even in those who underwent LPS showed further subnormal reduction of the average OCT-RNFL thickness which denotes a degree of ON fiber loss during the disease process (Fig. 1b). A big proportion of such patients had history of grade III and IV papilledema. This finding direct us to re-consider the ideal timing of LPS and avoid postponing which may results in irreversible visual affection. This observation is in accordance with the work of Hoffmann and colleagues 2018 [12] and Wall and colleagues 2017 [6] who stated that delayed affection of the central vision in IIH may result in initial misdiagnosis which may result in irreversible visual affection.

\section{Conclusion}

Newly diagnosed IIH patients' evaluation must be multimodality neuro-ophthalmological assessment based where papilledema grade, MD-VFE, and OCT-RNFL are valuable biomarkers of PVD while P100 VEP latency delay is a predictor of poor visual outcome and ONSD is an early indicator of elevated ICP regression after LPS surgery. On the other hand, increased BMI, high opening CSF pressure, and the presence of ANP, TVO EST, and/or transverse sinus stenosis during baseline assessment are not essentially predictors of subsequent IIH progression and need of LPS.

\section{Recommendations}

The management protocols of IIH need to be updated with the provision of evidence-based guidelines regarding the optimal medications, and indications of early surgical interference to ensure better outcomes. 


\section{Study strength}

The selection process is clear, and the studied sample was homogenous by exclusion of recurrent $\mathrm{IIH}$ patients. Multiple tests and maneuvers were done to insure multidisciplinary results which can be clinically applied.

\section{Limitations}

The use of only one modality for CSF shunting (lumbo-peritoneal shunts) and non-availability of other procedures like ON fenestration, ventriculo-peritoneal, or lumbo-pleural shunting to assess their values. Non-clarification of the effect of remote decrease in OCT-RNFL thickness on the initial treatment decisions which needs further studying.

\begin{abstract}
Abbreviations
ANP: Abducent nerve palsy; BMI: Body mass index; CSF: Cerebrospinal fluid; EST: Empty sella turcica; HPT: Headache onset-papilledema diagnosis time; ICP: Intracranial pressure; IIH: Idiopathic intracranial hypertension; LPS: Lumboperitoneal shunt; MD-VFE: Mean deviation of the visual field examination; OCT: Optical coherence tomography; ON: Optic nerve; ONSD: Optic nerve sheath diameter; PVD: Progressive visual deterioration; RNFL: Retinal nerve fiber layer; SD-OCT: Spectral domain optical coherence tomography; THISS: Tinnitus handicap inventory severity scale; TVO: Transient visual obscuration; VEP: Visual evoked potential; VFE: Visual field examination
\end{abstract}

\section{Acknowledgements}

We would like to thank the Neurology and Ophthalmology Departments Staff, Tanta University Hospitals for their great help in patients' selection, assessments, and follow-up.

\section{Funding}

No funding had been received.

\section{Availability of data and materials}

The datasets used and/or analyzed during the current study are available from the corresponding author on reasonable request.

\section{Authors' contributions}

WSB participated in the study's idea, design, patients' selection and examination, statistical and data analysis, CSF manometry, imaging interpretation, references collection, manuscript writing, revision, and final approval. YAH participated in study's idea and design, patients' assessment and inclusion, imaging interpretation, CSF manometry, data analysis, and critical revision of manuscript. MEME participated in patients' clinical examination, follow-up and registration, imaging interpretation, statistics, and manuscript writing. AFS participated in study's design, patients' collection, neuro-ophthalmological assessment, transorbital sonography and perimetry performance, statistical analysis, revision, and final approval. HAK participated in study's design, patients' inclusion and neuro-ophthalmological assessment, perimetry, OCT and VEP performance, data analysis, and critical revision of manuscript. All authors read and approved the final manuscript.

\section{Ethics approval and consent to participate}

- The manuscript was approved from The Research Ethics Committee and Quality Assurance Unit, Faculty of Medicine, Tanta University.

- The URL: http://tqac.tanta.edu.eg/new-tqac/

QualityAssuranceUnit@hotmail.com

- Approval Code: 31698/08/17.

- Name of the PI: Wafik Said Kamel El-Bahnasy.

- Name of the department: Neuropsychiatry.

- Type of the research: promotion research.

- Date of approval: July 2017.

- The study's protocol was approved by The Research Ethics Committee and Quality Assurance Unit, Faculty of Medicine, Tanta University. Participations were voluntary, informed consents were approved by all participants, and any possible risks were clarified.
Consent for publication

Not applicable.

\section{Competing interests}

The authors declare that they have no competing interests.

\section{Publisher's Note}

Springer Nature remains neutral with regard to jurisdictional claims in published maps and institutional affiliations.

Received: 31 December 2018 Accepted: 22 April 2019

Published online: 08 May 2019

References

1. Libien J, Kupersmith MJ, Blaner W, McDermott MP, Gaod S, Liua Y, et al. Role of vitamin a metabolism in $\mathrm{IH}$ : results from the idiopathic intracranial hypertension treatment trial. J Neurol Sci. 2017;372:78-84. https://doi.org/10. 1016/j.jns.2016.11.014.

2. Agarwal A, Vibha D, Prasad K, Bhatia R, Singh MB, Garg A, et al. Predictors of poor visual outcome in patients with idiopathic intracranial hypertension (IIH): an ambispective cohort study. Clin Neurol Neurosurg. 2017;159:13-8. https://doi.org/10.1016/j.clineuro.2017.05.009.

3. Miller NR. Non-neoplastic disorders mimicking brain tumors. Pseudotumor Cerebri. In: Winn HR, editor. Youmans \& Winn Neurological Surgery. 7th ed. Elsevier. 2017; 164:1352-65. https://lccn.loc.gov/2016035901

4. Smith SV, Friedman DI. The idiopathic intracranial hypertension treatment trial: a review of the outcomes. Headache. 2017;57(8):1303-10. https://doi. org/10.1111/head.13144.

5. Eidsvaag VA, Hansson HA, Heuser K, Nagelhus EA, Eide PK. Cerebral microvascular abnormalities in patients with idiopathic intracranial hypertension. Brain Res. 2018;1686:72-82. https://doi.org/10.1016/j.brainres. 2018.02.017

6. Wall M, Kupersmith MJ, Thurtell M, Moss HE, Moss EA, Auinger $P$, et al. The longitudinal idiopathic intracranial hypertension trial: outcomes from months 6-12. Am J Ophthalmol. 2017;176:102-7. https://doi.org/10.1016/j. ajo.2017.01.004

7. Olesen J, Bolay H, Charles A, Evers S, First M, Hershey A, et al. The international classification of headache disorders, $3^{\text {rd }}$ edition. Cephalalgia. 2018;38(1):1-211. https://doi.org/10.1177/0333102417738202.

8. Kishk NA, Ebraheim AM, Ashour AS, Badr NM, Eshra MA. Optic nerve sonographic examination to predict raised intracranial pressure in idiopathic intracranial hypertension: the cut-off points. Neuroradiol J. 2018;31(5):490-5. https://doi.org/10.1177/1971400918789385.

9. Bassi ST, Mohana KP. Optical coherence tomography in papilledema and pseudo-papilledema with and without optic nerve head drusen. Indian J Ophthalmol. 2014;62(12):1146-51. https://doi.org/10.4103/0301-4738.149136.

10. Odom JV, Bach M, Brigell M, Holder GE, McCulloch DL, Mizota A, et al. ISCEV standard for clinical visual evoked potentials: (2016 update). Doc Ophthalmol. 2016;133(1):1-9. https://doi.org/10.1007/s10633-016-9553-y.

11. Wall M, Kupersmith MJ, Kieburtz KD, Corbett JJ, Feldon SE, Friedman DI, et al. The idiopathic intracranial hypertension treatment trial. JAMA Neurol. 2014;71(6):693-701. https://doi.org/10.1001/jamaneurol.2014.133.

12. Hoffmann J, Mollan SP, Paemeleire K, Lampl C, Jensen RH, Sinclair AJ. European headache federation guideline on idiopathic intracranial hypertension. J. Headache Pain. 2018;19:93. https://doi.org/10.1186/s10194018-0919-2.

13. Mulroy E, Krishnan T, Best S, Anderson NE. Forgetting the fundoscope-a case of fulminant idiopathic intracranial hypertension causing rapid visual loss. J Clin Neurosci. 2018:50:108-10.

14. Berezovsky DE, Bruce BB, Vasseneix C, Peragallo JH, Newman NJ, Biousse V. Cerebrospinal fluid total protein in idiopathic intracranial hypertension. Neurol Sci. 2017;381:226-9. https://doi.org/10.1016/j.jns.2017.08.3264.

15. Markey KA, Mollan SP, Jensen RH, Sinclair AJ. Understanding idiopathic intracranial hypertension: mechanisms, management, and future directions. Lancet Neurol. 2016;15:78-91.

16. Fahmy IM, Abokrysha NT, Ahmed SM, El-Shebawy HM. Ultrasound evaluation of internal jugular valve incompetence (IJVI) in Egyptian patients with idiopathic intracranial hypertension (IIH). J Neurol Sci. 2016;360:18-22. https://doi.org/10.1016/j.jns.2015.11.034 
17. Biousse V. Idiopathic intracranial hypertension: diagnosis, monitoring and treatment. Rev Neurol. 2012;168:673-83. https://doi.org/10.1016/j.neurol. 2012.07.018.

18. Margeta MA, Buckley EG, El-Dairi MA. Low cerebrospinal fluid protein in prepubertal children with idiopathic intracranial hypertension. J AAPOS. 2015;19:135-9. https://doi.org/10.1016/j.jaapos.2015.01.006.

19. Galindo LM, Martínez RF, Rodrigo CF, de Miguel DF, Royo VP, García JP, JPG Inn, et al. Idiopathic intracranial hypertension: experience over 25 years and a management protocol. An Pediatr (Barc). 2017;87(2):78-86.

20. Bekerman I, Sigal T, Kimiagar I, Ben Ely A, Vaiman M. The quantitative evaluation of intracranial pressure by optic nerve sheath diameter/eye diameter CT measurement. Am J Emerg Med. 2016;34:2336-42. https://doi. org/10.1016/j.ajem.2016.08.045.

21. Liu D, Li Z, Zhang X, Zhao L, Jia J, Sun F, et al. Assessment of intracranial pressure with ultrasonographic retrobulbar optic nerve sheath diameter measurement. BMC Neurol. 2017;17(1):188-95. https://doi.org/10.1186/ s12883-017-0964-5.

22. Ang M, Tan ACS, Cheung CMG, Keane PA, Dolz-Marco R, Sng CCA, et al. Optical coherence tomography angiography: a review of current and future clinical applications. Graefes Arch Clin Exp Ophthalmol. 2018;256(2):237-45. https://doi.org/10.1007/s00417-017-3896-2.

23. Huang-Link YM, Al-Hawasi A, Oberwahrenbrock T, Jin YP. OCT measurements of optic nerve head changes in idiopathic intracranial hypertension. Clin Neurol Neurosurg. 2015;130:122-7. https://doi.org/10. 1016/j.clineuro.2014.12.021

24. Hartmann CJ, Klistorner A, Brandt AU, Schroeder K, Kolbe R, Cohn E, et al. Axonal damage in papilledema linked to idiopathic intracranial hypertension as revealed by multifocal visual evoked potentials. Clin Neurophysiol. 2015;126:2040-1. https://doi.org/10.1016/j.clinph.2014.12.014.

\section{Submit your manuscript to a SpringerOpen ${ }^{\circ}$ journal and benefit from:}

- Convenient online submission

- Rigorous peer review

- Open access: articles freely available online

High visibility within the field

- Retaining the copyright to your article

Submit your next manuscript at $\boldsymbol{\nabla}$ springeropen.com 